\title{
Analytical comparisons of standardized nasal cavity
}

\begin{abstract}
Human nasal cavities are unique and distinct when compared to each other. This causes difficulties in comparing the available information as there are no clear guidelines or standard procedures to adhere with. The creation of a standardized model is important to represent a certain population and will be beneficial for related fields of research. A standardized healthy female Malaysian nasal cavity was used as an initial step towards generalizing the bigger population. This is the first essential step towards solving inter-individual differences caused by geometrical variations among humans and the lack of universality from previous researches. In this study, critical comparisons were carried out to evaluate the standardized model with eight different models from previous works. This paper focuses on several aspects for comparisons such as cross sectional areas of the complete model, half model (right nasal cavity) and pressure drops obtained from computational analysis. The results show obvious differences as individual models used were obtained from different populations. This provides a new opportunity for a better understanding of the standardized model which will be a vital addition to the available models in literature.
\end{abstract}

Keyword: Comparisons; Cross sectional areas; Nasal cavity; Standardized model 